\title{
IAMJ
}

INTERNATIONAL AYURVEDIC MEDICAL JOURNAL

\section{A REVIEW ON THE PRAMEHAHARA DRAVYA OF KAIYADEVA NIGHANTU - REVIEW ARTICLE}

$\underline{\text { Vaishali }}^{1^{*}}$, Suguna Jyothy $\mathbf{P}^{2}$, Madhusudhan Rao $\mathbf{K}^{3}$

${ }^{1}$ P.G. Scholar, ${ }^{2}$ Professor \& HOD, ${ }^{3}$ Associate Professor

Department of Dravyaguna, Dr. N. R. S. Government Ayurvedic College, Vijayawada, India

\section{Corresponding Author: pittanivaishali@gmail.com}

\section{https://doi.org/10.46607/iamj12p5062021}

(Published online: September 2021)

Open Access

(C) International Ayurvedic Medical Journal, India 2021

Article Received: 18/08/2021 - Peer Reviewed: 12/09/2021 - Accepted for Publication: 15/09/2021

Check for updates

\begin{abstract}
Prameha foremost in lifestyle disorders is mentioned among the Astamahagadas in Ayurvedic classics. Diabetes can be co-related with Prameha which is characterised by impaired blood glucose metabolism. Prameha according to Ayurvedic Literature is the Santarpana Janya Vyadhi, it is mainly characterised by Prabhutha avila mutrata (Polyuria), Paani pada daha (burning sensation over foot), Trishna (Polydipsia). The Prevalence of this disease is increasing worldwide making India the rising capital of Diabetes. The global diabetes prevalence in 2019 was estimated to be $9.3 \%$ (463 million people), rising to 10.2\% (578 million) by 2030 and 10.9\% (700 million) by 2045 . The need of the hour is to find efficient management in the alleviation of Prameha. Kaiyadeva Nighantu also known as Pathya-Apathya vibhodaka is the classical text which describes the dietic benefits in ailments. The work of Kaiyadeva Nighantu has been divided into Aushadha, Ahara and Vihara Vargas. There are nine varga available in this Nighantu and in total there are 514 drugs in Aushadhivarga, among them, 53 dravyas are having Prameha hara property. These drugs will help both physicians and researchers in their respective fields, either for the treatment of Prameha or in the field of research to explore the unknown. The present study has been done to analyse potent herbal remedies in the management of Prameha.
\end{abstract}

Keywords: Prameha, Kaiyadeva Nighantu, Diabetes, Herbs. 


\section{INTRODUCTION}

India is regarded as a treasure trove of herbs in the world. The proper and judicious use of herbs is often successful in the treatment of illness. In the present scenario, sedentary lifestyle and stressful mental conditions are running down too many distressing diseases, among them, Prameha is foremost in lifestyle disorders, and it has been described by our acharyas as one among the Astamahagadas. "Prakarshen Prabhutam- Prachuram varamvaram va mehati mutratyaga karoti yasmin roge sa pramehah"- (Madhava Nidhana $)^{1}$. The features of Prameha mentioned in Ayurveda can be compared with Diabetes. Diabetes Mellitus, a multifaceted disease refers to a group of common metabolic disorders that share the phenotype of Hyperglycaemia. Prameha according to Ayurvedic Literature is the Santarpana Janya Vyadhi, characterised by the symptoms like Prabhutha avila mutrata, Paani pada daha, Trishna. Diabetes mellitus is an endocrine disorder wherein the production of insulin is disturbed. The improper regulation of glucose metabolism leads to the clinical presentation of symptoms like polyuria, polydipsia, polyphagia, increased blood sugar, urine sugar levels, sleeplessness, hormonal imbalances and many other associated anomalies. The incidence of Diabetes has shown an alarming increase around the world. According to W.H.O. the global prevalence of diabetes is estimated to be $\mathbf{3 6 6}$ million by 2030. The global diabetic's ratio is growing devastatingly, so the need of the hour is to find effective ways in combating the disease.

Kaiyadeva Nighantu is one of the known compendiums of Ayurvedic texts. Kaiyadeva Nighantu is also known as Pathya Apathya Vibhodaka. The author of the original text is Acharaya Kaiyadeva belonging to $14^{\text {th }}$ Century A.D. and has given utmost importance to maintaining normal health and preventing diseases. Keeping this in view the author has planned his work as Aushadha, Ahara and Vihara Vargas. The work has been compiled with regards to the knowledge of plants based on their Paryaya and Gunadharma. The Nighantu has been classified into nine vargas. The present text has been taken for the study of Prameha hara dravyas. The aim and objective of the study are to analyse the Prameha hara dravyas mentioned in Kaiyadeva Nighantu following the principles of Ayurveda in understanding them.

\section{Materials and Methods}

- Kaiyadeva nighantu (Pathya Apathya Vibodhakah) edited and translated to Hindi by Prof. Priyavata Sharma Ji and Dr Guru Prasada Sharma Ji has been taken as the base for the study.

- The published work on journals and web pages are consulted for review of the Kaiyadeva Nighantu and Prameha hara dravyas mentioned in other texts for better understanding.

- The Nighantu has been searched for the term Prameha hara and the drugs mentioned to have the said property were listed out. The properties mentioned for the list of the plants are tabulated and critically analysed on the principles of Dravyaguna to identify the most probable properties of all the Prameha hara dravyas.

\section{Observations and Results}

From the observation, it was identified that 53 dravyas are mentioned to have been included in the Pramehahara. The list of the dravyas has been enlisted in Table no: 1 below.

Table 1: Showing the list of the plants having the Pramehahara property

\begin{tabular}{|c|c|c|c|c|c|c|c|c|}
\hline S.no. & $\begin{array}{l}\text { Name of the } \\
\text { drug }\end{array}$ & $\begin{array}{l}\text { Botanical } \\
\text { name } \\
\text { Family }\end{array}$ & Parts used & Rasa & Guna & Virya & Vipaka & $\begin{array}{l}\text { Dosha } \\
\text { Karma }\end{array}$ \\
\hline 1. & Guduchi & $\begin{array}{l}\text { Tinospora } \\
\text { cordifolia } \\
\text { Willd. } \\
\text { Menisperma- } \\
\text { ceae }\end{array}$ & $\begin{array}{l}\text { Kanda }(\text { St. }), \\
\text { Patra }(\text { L. })\end{array}$ & $\begin{array}{l}\text { Tikta, } \\
\text { Kashaya, } \\
\text { Katu }\end{array}$ & Laghu & Ushna & Madhur & $\begin{array}{l}\text { Tridosha } \\
\text { hara }\end{array}$ \\
\hline
\end{tabular}




\begin{tabular}{|c|c|c|c|c|c|c|c|c|}
\hline 2. & Vasa & $\begin{array}{l}\text { Adhatoda va- } \\
\text { sica Nees. } \\
\text { Acanthaceae }\end{array}$ & Patra (L.) & $\begin{array}{l}\text { Kashaya, } \\
\text { Tikta }\end{array}$ & Laghu & Sita & Katu & KP hara \\
\hline 3. & Saliparni & $\begin{array}{l}\text { Desmodium } \\
\text { gangeticum } \\
\text { D.C. } \\
\text { Leguminosae }\end{array}$ & Mula (Rt.) & $\begin{array}{l}\text { Madhura, } \\
\text { Tikta }\end{array}$ & Guru & Ushna & Madhur & $\begin{array}{l}\text { Tridosha } \\
\text { hara }\end{array}$ \\
\hline 4. & $\begin{array}{l}\text { Sveta Kan- } \\
\text { takari }\end{array}$ & $\begin{array}{l}\text { Solanum } \\
\text { xantho- } \\
\text { carpum } \\
\text { Schrad and } \\
\text { Wendl. }\end{array}$ & Puspha(fl.) & Katu, Tikta & $\begin{array}{l}\text { Laghu, } \\
\text { Ruksha }\end{array}$ & Ushna & Katu & KV hara \\
\hline 5. & Goksura & $\begin{array}{l}\text { Tribulus ter- } \\
\text { restris Linn. } \\
\text { Zygophyl- } \\
\text { laceae }\end{array}$ & $\begin{array}{l}\text { Panca-anga } \\
\text { (whole plant) }\end{array}$ & Madhura & $\begin{array}{l}\text { Guru, } \\
\text { Snigdha }\end{array}$ & Sita & Madhur & $\begin{array}{l}\text { Tridosa } \\
\text { hara }\end{array}$ \\
\hline 6. & Eranda & $\begin{array}{l}\text { Ricinus com- } \\
\text { munis Linn. } \\
\text { Euphorbia- } \\
\text { ceae }\end{array}$ & $\operatorname{Mula}(R t)$. & Madhura & Guru & Ushna & Katu & $\begin{array}{l}\text { Tridosha } \\
\text { hara }\end{array}$ \\
\hline 7. & Haritaki & $\begin{array}{l}\text { Terminalia } \\
\text { chebula Retz. } \\
\text { Combreta- } \\
\text { ceae }\end{array}$ & Phala(Fr.) & $\begin{array}{l}\text { Kashaya } \\
\text { Pradhana } \\
\text { Panca Rasa } \\
\text { Alavana }\end{array}$ & $\begin{array}{l}\text { Ruksha, } \\
\text { Laghu }\end{array}$ & Ushna & Madhur & $\begin{array}{l}\text { Tridosha } \\
\text { hara }\end{array}$ \\
\hline 8. & Kadali & $\begin{array}{l}\text { Musa sapien- } \\
\text { tum Linn. } \\
\text { Musaceae }\end{array}$ & $\begin{array}{l}\text { Kadali toya, ta- } \\
\text { runa kadali } \\
\text { phala, Krishna } \\
\text { kadali } \\
\text { phala }(F r .)\end{array}$ & $\begin{array}{l}\text { Tikta, } \\
\text { Kashaya }\end{array}$ & $\begin{array}{l}\text { Ruksha, } \\
\text { Laghu }\end{array}$ & Sita & Madhur & $\begin{array}{l}\text { Pitta } \\
\text { hara }\end{array}$ \\
\hline 9. & Amra & $\begin{array}{l}\text { Mangifera } \\
\text { indica Linn. } \\
\text { Anacardi- } \\
\text { aceae }\end{array}$ & Pupsha (fl.) & Kashaya & Ruksha & Sita & Katu & KP hara \\
\hline 10. & Amlika & $\begin{array}{l}\text { Tamarindus } \\
\text { indicus } \\
\text { Leguminosae }\end{array}$ & Puspha (fl.) & $\begin{array}{l}\text { Madhura, } \\
\text { Kashaya, } \\
\text { Amla }\end{array}$ & Laghu & Ushna & Katu & KV hara \\
\hline 11. & Kapitta & $\begin{array}{l}\text { Feronia ele- } \\
\text { phantum } \\
\text { Correa } \\
\text { Rutaceae }\end{array}$ & Patra (L.) & $\begin{array}{l}\text { Tikta, } \\
\text { Kashaya }\end{array}$ & Tikshna & Ushna & Katu & K hara \\
\hline 12. & Udumbara & $\begin{array}{l}\text { Ficus glom- } \\
\text { erata Roxb. } \\
\text { Moraceae }\end{array}$ & Pakwa phala & Madhura & Guru & Sita & Madhur & K kara \\
\hline 13. & Tuvarka & $\begin{array}{l}\text { Hydnocarpus } \\
\text { wightiana } \\
\text { Blume. } \\
\text { Flacour- } \\
\text { tiaceae }\end{array}$ & Phala(Fr.) & Kashaya & $\begin{array}{l}\text { Snigdha, } \\
\text { Guru }\end{array}$ & Ushna & Katu & K hara \\
\hline 14. & Patola & $\begin{array}{l}\text { Trichosan- } \\
\text { thes dioica } \\
\text { Roxb. } \\
\text { Cucurbita- } \\
\text { ceae }\end{array}$ & Phala(Fr.) & Katu, Tikta & $\begin{array}{l}\text { Laghu, } \\
\text { Snigdha }\end{array}$ & Ushna & Madhur & $\begin{array}{l}\text { Tridosha } \\
\text { hara }\end{array}$ \\
\hline
\end{tabular}




\begin{tabular}{|c|c|c|c|c|c|c|c|c|}
\hline 15. & Kosataki & $\begin{array}{l}\text { Luffa acutan- } \\
\text { gular Roxb. } \\
\text { Cucurbita- } \\
\text { ceae }\end{array}$ & Phala(Fr.) & Tikta & $\begin{array}{l}\text { Tikshna, } \\
\text { Laghu, } \\
\text { Ruksha }\end{array}$ & Sita & Katu & $\begin{array}{l}\text { Tridosha } \\
\text { hara }\end{array}$ \\
\hline 16. & Karavellaka & $\begin{array}{l}\text { Momordica } \\
\text { charantia } \\
\text { Linn. } \\
\text { Cucurbita- } \\
\text { ceae }\end{array}$ & Phala(Fr.) & $\begin{array}{l}\text { Tikta, } \\
\text { Katu }\end{array}$ & Laghu & Ushna & Katu & $\begin{array}{l}\text { Kapha } \\
\text { hara }\end{array}$ \\
\hline 17. & Karkotaki & $\begin{array}{l}\text { Momordica } \\
\text { dioica Roxb. } \\
\text { Cucurbita- } \\
\text { ceae }\end{array}$ & Phala(Fr.) & $\begin{array}{l}\text { Tikta, } \\
\text { Madhura }\end{array}$ & Laghu & Ushna & Madhur & $\begin{array}{l}\text { Tridosha } \\
\text { hara }\end{array}$ \\
\hline 18. & Visamushti & $\begin{array}{l}\text { Strychnos } \\
\text { nuxvomica } \\
\text { Loganiaceae }\end{array}$ & Phala(Fr.) & $\begin{array}{l}\text { Katu tikta, } \\
\text { Kashaya }\end{array}$ & Laghu & Ushna & Katu & K hara \\
\hline 19. & Brhat launika & $\begin{array}{l}\text { Portulaca } \\
\text { oleracea } \\
\text { Linn. } \\
\text { Portulaca }\end{array}$ & $\begin{array}{l}\text { Panca-anga } \\
\text { (wholeplant) }\end{array}$ & Amla & Ruksha & Ushna & Katu & $\begin{array}{l}\text { V hara, } \\
K P \\
\text { vardaka }\end{array}$ \\
\hline 20 . & $\begin{array}{l}\text { Kuchelika } \\
\text { (brhat patha) }\end{array}$ & $\begin{array}{l}\text { Cyclea pel- } \\
\text { tate } \\
\text { Menisperma- } \\
\text { ceae }\end{array}$ & Patra (L.) & $\begin{array}{l}\text { Tikta, } \\
\text { Kashaya, } \\
\text { Madhura }\end{array}$ & $\begin{array}{l}\text { Ruksha, } \\
\text { guru }\end{array}$ & Sita & Madhur & KP hara \\
\hline 21. & Sunisanka & $\begin{array}{l}\text { Marsilea } \\
\text { minuta Linn. } \\
\text { Rhizocarpeae }\end{array}$ & $\begin{array}{l}\text { Panca-anga } \\
\text { (whole plant) }\end{array}$ & $\begin{array}{l}\text { Madhura, } \\
\text { Kashaya }\end{array}$ & $\begin{array}{l}\text { Laghu, } \\
\text { ruksha }\end{array}$ & Sita & Katu & $\begin{array}{l}\text { Tridosha } \\
\text { hara }\end{array}$ \\
\hline 22. & Cakramardha & $\begin{array}{l}\text { Cassia tora } \\
\text { Linn. } \\
\text { Leguminosae }\end{array}$ & Phala (Fr.) & $\begin{array}{l}\text { Katu, } \\
\text { Madhura, } \\
\text { Lavana }\end{array}$ & $\begin{array}{l}\text { Laghu, } \\
\text { ruksha }\end{array}$ & Ushna & Katu & $\begin{array}{l}\text { VP } \\
\text { vardaka }\end{array}$ \\
\hline 23. & Bakuchi & $\begin{array}{l}\text { Psoralea cor- } \\
\text { ylifolia Linn. } \\
\text { Leguminosae }\end{array}$ & - & $\begin{array}{l}\text { Kashaya, } \\
\text { Tikta, } \\
\text { Madhura }\end{array}$ & $\begin{array}{l}\text { Laghu, } \\
\text { ruksha }\end{array}$ & Sita & Katu & K hara \\
\hline 24. & Kakamachi & $\begin{array}{l}\text { Solanum } \\
\text { nigrum Linn. } \\
\text { Solanaceae }\end{array}$ & $\begin{array}{l}\text { Panca-anga } \\
\text { (whole plant) }\end{array}$ & $\begin{array}{l}\text { Katu, } \\
\text { Tikta }\end{array}$ & $\begin{array}{l}\text { Laghu, } \\
\text { snigdha }\end{array}$ & Ushna & Katu & $\begin{array}{l}\text { Tridosha } \\
\text { hara }\end{array}$ \\
\hline 25. & Gojihva & $\begin{array}{l}\text { Elephantopus } \\
\text { scaber Linn. } \\
\text { Compositae }\end{array}$ & - & $\begin{array}{l}\text { Kashaya, } \\
\text { Tikta, } \\
\text { Madhura }\end{array}$ & Laghu & Sita & Madhur & KP hara \\
\hline 26. & Mesasringi & $\begin{array}{l}\text { Gymnema } \\
\text { sylvestre } \\
\text { Asclepiada- } \\
\text { ceae }\end{array}$ & Phala (Fr.) & Tikta & $\begin{array}{l}\text { Laghu, } \\
\text { Ruksha }\end{array}$ & Ushna & Katu & KP hara \\
\hline 27. & Murva & $\begin{array}{l}\text { Marsdenia } \\
\text { tenacissima } \\
\text { W.\& A. } \\
\text { Asclepiada- } \\
\text { ceae }\end{array}$ & Mula (Rt.) & $\begin{array}{l}\text { Madhura, } \\
\text { Tikta }\end{array}$ & Guru & Ushna & Madhur & $\begin{array}{l}\text { Tridosha } \\
\text { hara }\end{array}$ \\
\hline 28. & Bijaka & $\begin{array}{l}\text { Pterocarpus } \\
\text { marsupium } \\
\text { Leguminosae }\end{array}$ & Sara (Hr.wd.) & $\begin{array}{l}\text { Kashaya, } \\
\text { Katu }\end{array}$ & $\begin{array}{l}\text { Laghu, } \\
\text { Ruksha }\end{array}$ & Anushna & Katu & KP hara \\
\hline 29. & Tinisa & $\begin{array}{l}\text { Ougeinia dal- } \\
\text { bergiodes, le- } \\
\text { guminaceae }\end{array}$ & Sara (Hr.wd.) & Kashaya & Ruksha & Ushna & Katu & P hara \\
\hline
\end{tabular}




\begin{tabular}{|c|c|c|c|c|c|c|c|c|}
\hline 30. & Arjuna & $\begin{array}{l}\text { Terminalia } \\
\text { arjuna com- } \\
\text { bretaceae }\end{array}$ & $\begin{array}{l}\text { Kanda twak } \\
\text { (St.br.) }\end{array}$ & Kashaya & $\begin{array}{l}\text { Laghu, } \\
\text { Ruksha }\end{array}$ & Sita & Katu & PK hara \\
\hline 31. & Kadhira & $\begin{array}{l}\text { Acacia cate- } \\
\text { chu } \\
\text { Leguminosae }\end{array}$ & Sara (Hr.wd.) & $\begin{array}{l}\text { Tikta, } \\
\text { kashaya }\end{array}$ & $\begin{array}{l}\text { Laghu, } \\
\text { Ruksha }\end{array}$ & Sita & Katu & KP hara \\
\hline 32. & Palasha & $\begin{array}{l}\text { Butea fron- } \\
\text { dose } \\
\text { Leguminosae }\end{array}$ & Puspha (fl.) & $\begin{array}{l}\text { Madhura, } \\
\text { Tikta, } \\
\text { Kashaya }\end{array}$ & Ruksha, & Sita & Katu & KP hara \\
\hline 33. & Dhava & $\begin{array}{l}\text { Anogeissus } \\
\text { latifolia } \\
\text { Combreta- } \\
\text { ceae }\end{array}$ & $\begin{array}{l}\text { Kanda twak } \\
\text { (St.br.) }\end{array}$ & $\begin{array}{l}\text { Kashaya, } \\
\text { Madhura }\end{array}$ & $\begin{array}{l}\text { Ruksha, } \\
\text { laghu }\end{array}$ & Sita & Katu & KP hara \\
\hline 34. & Aswakarna & $\begin{array}{l}\text { Dipterocar- } \\
\text { pus alatus } \\
\text { Dipterocar- } \\
\text { paceae }\end{array}$ & $\begin{array}{l}\text { Kanda twak } \\
\text { (St.br.) }\end{array}$ & $\begin{array}{l}\text { Katu, Tikta, } \\
\text { Kashaya }\end{array}$ & $\begin{array}{l}\text { Laghu, } \\
\text { Ruksha }\end{array}$ & Ushna & Katu & K hara \\
\hline 35. & Mochaka & $\begin{array}{l}\text { Schrebera } \\
\text { swietenioides } \\
\text { Oleaceae }\end{array}$ & $\begin{array}{l}\text { Kanda twak } \\
\text { (St.br.) }\end{array}$ & $\begin{array}{l}\text { Katu, } \\
\text { Tikta }\end{array}$ & Tikshna & Ushna & Katu & $\begin{array}{l}\text { KP sa- } \\
m a k a\end{array}$ \\
\hline 36. & Nimba & $\begin{array}{l}\text { Azadiracta } \\
\text { indica } \\
\text { Meliaceae }\end{array}$ & $\begin{array}{l}\text { Kanda twak } \\
\text { (St.br.) }\end{array}$ & Tikta & Laghu & Sita & Katu & $\begin{array}{l}\text { KP sa- } \\
\text { maka }\end{array}$ \\
\hline 37. & Kiratatikta & $\begin{array}{l}\text { Swertia chi- } \\
\text { rata } \\
\text { Gentianaceae }\end{array}$ & $\begin{array}{l}\text { Panca-anga } \\
\text { (wholeplant) }\end{array}$ & Tikta & $\begin{array}{l}\text { Ruksha, } \\
\text { Laghu, } \\
\text { Sara }\end{array}$ & Sita & Katu & $\begin{array}{l}\text { KP sa- } \\
\text { maka }\end{array}$ \\
\hline 38. & Sehandu & $\begin{array}{l}\text { Euphorbia } \\
\text { nerifolia } \\
\text { Euphorbia- } \\
\text { ceae }\end{array}$ & Patra (L.) & $\begin{array}{l}\text { Katu, } \\
\text { Tikta }\end{array}$ & $\begin{array}{l}\text { Guru, Tik- } \\
\text { shna }\end{array}$ & Ushna & Katu & $V$ samaka \\
\hline 39. & Aragwadha & $\begin{array}{l}\text { Cassia fistula } \\
\text { Leguminosea }\end{array}$ & $\begin{array}{l}\text { Phalamajja } \\
\text { (Fr.pulp) }\end{array}$ & $\begin{array}{l}\text { Tikta, } \\
\text { Madhura }\end{array}$ & Guru & Sita & Madhur & $\begin{array}{l}\text { Tridosha } \\
\text { samaka }\end{array}$ \\
\hline 40. & Kampillaka & $\begin{array}{l}\text { Mallotus } \\
\text { phillippinen- } \\
\text { sis } \\
\text { Euphorbia- } \\
\text { ceae }\end{array}$ & Phalarajo & Katu & $\begin{array}{l}\text { Laghu, } \\
\text { Ruksha, } \\
\text { Tikshna }\end{array}$ & Ushna & Katu & $\begin{array}{l}K V \\
\text { nasaka }\end{array}$ \\
\hline 41. & Karanjika & $\begin{array}{l}\text { Caesalpinia } \\
\text { decapetala } \\
\text { Caesalpinoi- } \\
\text { dae }\end{array}$ & Kanda (St.) & $\begin{array}{l}\text { Katu, Tikta, } \\
\text { Kashaya }\end{array}$ & $\begin{array}{l}\text { Laghu } \\
\text { Ruksha }\end{array}$ & Ushna & Katu & $\begin{array}{l}\text { KV sa- } \\
\text { maka }\end{array}$ \\
\hline 42. & Avartaki & $\begin{array}{l}\text { Cassia auric- } \\
\text { ulata } \\
\text { Leguminosae }\end{array}$ & $\begin{array}{l}\text { Pupsha, Apakwa } \\
\text { Phala, Bija }\end{array}$ & $\begin{array}{l}\text { Tikta, } \\
\text { Kashaya }\end{array}$ & $\begin{array}{l}\text { Sara, } \\
\text { Laghu } \\
\text { Ruksha }\end{array}$ & Sita & Katu & $\begin{array}{l}\text { KP sa- } \\
\text { maka }\end{array}$ \\
\hline 43. & Indravaruni & $\begin{array}{l}\text { Citrullus col- } \\
\text { ocynthis } \\
\text { Cucurbita- } \\
\text { ceae }\end{array}$ & Phala (Fr.) & Tikta, Katu & Laghu & Ushna & Katu & $\begin{array}{l}P K \\
\text { nasaka }\end{array}$ \\
\hline 44. & Haridra & $\begin{array}{l}\text { Curcuma } \\
\text { longa } \\
\text { Zingibera- } \\
\text { ceae }\end{array}$ & $\operatorname{Kanda}(R z)$. & Tikta, Katu & Ruksha & Ushna & Katu & $\begin{array}{l}\text { KP } \\
\text { nasaka }\end{array}$ \\
\hline 45. & Katuki & $\begin{array}{l}\text { Picchoriza } \\
\text { kurroa }\end{array}$ & $\operatorname{Kanda}(R z)$. & Tikta, Katu & $\begin{array}{l}\text { Ruksha, } \\
\text { Laghu }\end{array}$ & Sita & Katu & $\begin{array}{l}\text { KP sa- } \\
\text { maka }\end{array}$ \\
\hline
\end{tabular}




\begin{tabular}{|c|c|c|c|c|c|c|c|c|}
\hline & & $\begin{array}{l}\text { Scrophu- } \\
\text { laceae }\end{array}$ & & & & & & \\
\hline 46. & Katphala & $\begin{array}{l}\text { Myrica nagi } \\
\text { Thunb. } \\
\text { Myricaceae }\end{array}$ & $\begin{array}{l}\text { Kanda twak } \\
(\text { St.br.) }\end{array}$ & $\begin{array}{l}\text { Katu, Tikta, } \\
\text { Kashaya }\end{array}$ & $\begin{array}{l}\text { Laghu, } \\
\text { Tikshna }\end{array}$ & Ushna & Katu & $\begin{array}{l}\text { KV } \\
\text { nasaka }\end{array}$ \\
\hline 47. & Pashanabedha & $\begin{array}{l}\text { Bergenia lig- } \\
\text { ulate } \\
\text { Saxifraga- } \\
\text { ceae }\end{array}$ & $\begin{array}{l}\text { Panca-anga } \\
\text { (whole-plant) }\end{array}$ & $\begin{array}{l}\text { Kashaya, } \\
\text { Tikta }\end{array}$ & Laghu & Sita & Katu & $\begin{array}{l}\text { KV } \\
\text { nasaka }\end{array}$ \\
\hline 48. & Vidanga & $\begin{array}{l}\text { Embelia } \\
\text { ribes } \\
\text { Myrsinaceae }\end{array}$ & Phala (Fr.) & Katu, Tikta & $\begin{array}{l}\text { Ruksha, } \\
\text { Laghu }\end{array}$ & Ushna & Katu & $\begin{array}{l}\text { VK } \\
\text { nasaka }\end{array}$ \\
\hline 49. & Pippali & $\begin{array}{l}\text { Piper longum } \\
\text { Piperaceae }\end{array}$ & Phala (Fr.) & Katu & $\begin{array}{l}\text { Laghu } \\
\text { Snigdha }\end{array}$ & Anushna & Madhur & KV hara \\
\hline 50. & Lasuna & $\begin{array}{l}\text { Allium sa- } \\
\text { tivum } \\
\text { Liliaceae }\end{array}$ & Kanda(bulb) & $\begin{array}{l}\text { Kashaya, } \\
\text { Madhura }\end{array}$ & $\begin{array}{l}\text { Guru, } \\
\text { Snigdha, } \\
\text { Sara, Tik- } \\
\text { shna }\end{array}$ & Anushna & Katu & KV hara \\
\hline 51. & Vetraka & $\begin{array}{l}\text { Calamus ten- } \\
\text { uis } \\
\text { Palmaceae }\end{array}$ & Phala (Fr.) & Amla & Laghu & Sita & Katu & KP hara \\
\hline 52. & Devadaru & $\begin{array}{l}\text { Cedrus deo- } \\
\text { dara } \\
\text { Pinaceae }\end{array}$ & $\begin{array}{l}\text { kanda twak } \\
(H r . w d .)\end{array}$ & Tikta, Katu & $\begin{array}{l}\text { Laghu, } \\
\text { Snigdha }\end{array}$ & Ushna & Katu & KV hara \\
\hline 53. & Manjistha & $\begin{array}{l}\text { Rubia cordi- } \\
\text { folia } \\
\text { Rubiaceae }\end{array}$ & Mula (Rt.) & $\begin{array}{l}\text { Madhura, } \\
\text { Tikta, } \\
\text { Kashaya }\end{array}$ & Guru & Ushna & Katu & $\begin{array}{l}\text { KP sa- } \\
\text { maka }\end{array}$ \\
\hline
\end{tabular}

\section{Results:}

- From the above-mentioned table, 53 drugs have been identified and their properties have been enlisted in Table no 1. The drugs are analysed based on their Rasa, Guna, Virya, Vipaka, and Karma on Dosha.

- Based on the Rasa among the 53 dravyas identified 17 dravyas have Tikta rasa, 12 dravyas have Kashaya rasa, 13 dravyas have Katu rasa, dravyas have 9 Madhura rasa, 2 dravyas have Amla rasa respectively.

- Based on the Guna of the dravyas it was observed that among 53 dravyas, 34 dravyas have Laghu guna, 24 dravyas have Ruksha guna, 11 dravyas have Guru guna, 7 dravyas had Snigdha and Tikshna gunas respectively.
- Based on the Virya of the identified dravyas it was observed that 29 dravyas are Ushna virya while 21 dravyas have Sita Virya and 03 dravya is having Anushna Virya.

- Based on the Vipaka of dravyas identified it was observed that among 53 dravyas 40 dravyas are Katu Vipaka, 13 have Madhura Vipaka respectively.

- Based on the Karma it has been observed that out of 53 dravyas 02 dravyas are Vatahara, 01 Pittahara, 06 dravyas are Kaphahara, 10 dravyas are Kaphavatahara, 21 dravyas are Kaphapittahara, and 11 dravyas are Tridosha hara respectively.

- The majority of the dravyas enlisted above have phala as a useful part. 


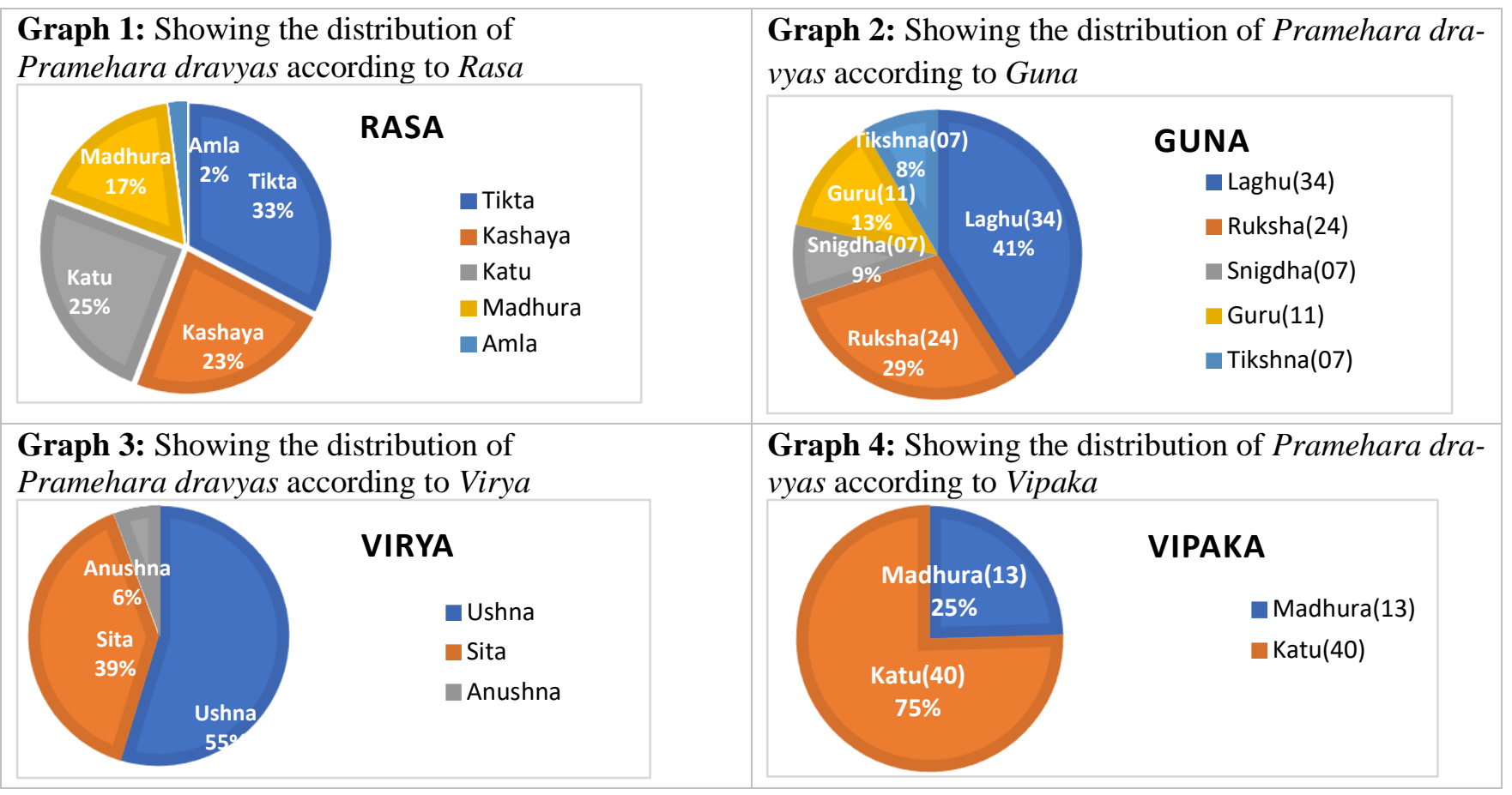

Graph 5: Showing the distribution of Pramehara dravyas according to Dosha Karma

\section{DISCUSSION}

Prameha according to Ayurveda is Santarpajanya vyadhi caused by improper diets, sedentary lifestyle, and associated metabolic disorders. The causative factors involved vitiates the Kapha dosha which further leads to vitiation of Vata and Pitta doshas, causing the involvement of Tridosha in the disease manifestation. The vitiated doshas further disturb the dhatus and malas expelling them out of the body by various means. Prameha is a multifactorial disease with many symptoms like sweetness in the mouth, numbness and burning sensation in hands \& feet's, dryness of mouth, palate and throat, disturbed sleep patterns. The prime symptoms of all the types of Prameha include increased quality and turbidity of urine, sweetness of

\section{KARMA}

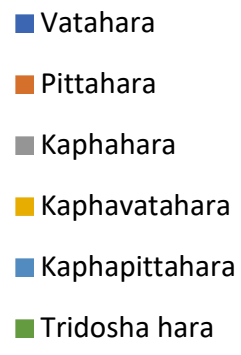

urine (mutramadhuryata), urine resembles like honey (madhuavivameha), raised sweetness of the whole body (madhuryaschcha tanorath). Since the disease is Kaphapradhan Tridoshaja so, we need potent dravyas which act on Kapha dosha as well balances Vata and Pitta dosha respectively.

In the present study of Prameha Hara dravyas mentioned in the Kaiyadeva Nighantu, it was found that there are potent herbal drugs having potentials in the management of Prameha. The entire Ayurvedic texts have been written on their principles and constant observations. Principles like Panchamahabhuta siddhanta, Tridosha, Samanya-vishesha, principles of sodhana and samana play a vital role in the management of ailments. Here, the dravyas have been analysed for 
their therapeutic action on Prameha based on its properties and action keeping these basic Siddhantas in view.

Generally, all the three doshas, which are involved in genesis of Prameha, affect the constituents such as Meda, Rakta, Sukra, Ambu, Vasa, Lasika, Majja, Rasa, Ojas. Dosha which enters the Vasti contaminates urine and produces Prameha. Classically Prameha has been enumerated as 20, among these 10 being Kaphaja Prameha caused due predominance of Kapha dosha along with the amalgamation of vitiated Vata, Pitta, and Meha. 6 types of Pittaja Prameha caused due to amalgamation of Tridosha along with Rakta and Meha as Dushiyas. 4 types of Vataja Prameha caused due to amalgamation with all the Dushiyas leads to the development of Madhumeha.

The majority of drugs mentioned above have Tikta, Kashaya rasa which has Sodhana and Sthambana action respectively. Also, the above rasa pacifies Kapha dosha which is the cause for the initiation of the disease. The Dravyas mentioned here mostly have Laghu and Ruksha guna which acts as Kapha samaka, furthermost of the drugs have Ushna virya and Katu vipaka because of its Ushna virya it acts as Kaphavata samaka and because of its Katu Vipaka it acts as Baddhavinmutra which aids in the management of Prameha. Few drugs have Madhura rasa, Madhura Vipaka and Sita virya which acts as Pitta Samaka and helps in the nourishment of depleted doshas.

\section{CONCLUSION}

Hence, from the above study, it can be concluded that dravyas having Laghu, Ruksha Gunas, Tikta, Kashaya rasa Pradhana, Katu Vipaka, and Ushna Virya will have better Pramehahara properties. The drugs which are mentioned here and having similar properties can be clinically tried to achieve the Pramehahara effect.

\section{REFERENCES}

1. Dr. Kanjiv lochan, Madhava Nidhana withmadhukosa by Vijayaraksita \& Srikanthadatta; Chaukhambha Publication, Varanasi

2. Prof. Priyavata Sharma \& Dr. Guru Prasada Sharma, Kaiyadeva-nighantu Hindi translation, Chaukhambha Publication, Varanasi; first edition (1979), Aushadivarga, 1/12; pgno:6; 1/1; pgno:5 1/46; pgno:12; 1/56; pgno:13; 1/70; pgno:16, 1/116; pgno:24, 1/225; pgno:45, 1/285;287,290; pgno:55-56, 1/337,338; pgno:63-64, 1/364; pgno:68, 1/421; pgno:75, 1/430; pgno:76, 1/504; pgno:92, 1/567; pgno:103, 1/572; pgno:104, 1/590; pgno:108; 1/599; pgno:109, 1/602; pgno:110, 1/648; pgno:119, 1/648; pgno:119, 1/691; pgno:125, 1/697; pgno:127, 1/701; pgno:130, 1/707; pgno:131, 1/711; pgno:131, 1/734; pgno:136, 1/739; pgno:136, 1/786; pgno:145, 1/813; pgno:151, 1/816; pgno:152, 1/820; pgno:153; 1/824; pgno:153, 1/835; pgno:155, 1/837; pgno:156, 1/845; pgno:157, 1/876; pgno:162, 1/880; pgno:163, 1/890; pgno:165, 1/918; pgno:170; 1/944; pgno:174, 1/949; pgno:175; 1/970; pgno:179; 1/1000; pgno:185; 1/1028; pgno:189, 1/1115; pgno:206; 1/1124; pgno:208; 1/1138; pgno:210; 1/1146; pgno:212; 1/1149; pgno:212, 1/1168; pgno:215; 1/1121; pgno:225; 1/1252; pgno:231; 1/1310; pgno:242; 1/1426; pgno:264

\section{Source of Support: Nil Conflict of Interest: None Declared}

How to cite this URL: Vaishali et al: A Review On The Pramehahara Dravya Of Kaiyadeva Nighantu - Review Article. International Ayurvedic Medical Journal \{online\} 2021 \{cited September 2021\} Available from: http://www.iamj.in/posts/images/upload/3122_3129.pdf 